\title{
Glass-break detector using accelerometer
}

\author{
Václav Mach, ", Jan Valouch, and Milan Adámek \\ Tomas Bata University in Zlín, Faculty of Applied Informatics, Nad Stráněmi 4511, 76005 Zlín, Czech Republic
}

\begin{abstract}
The aim of this paper is to summarize indoor detectors for peripheral intrusion detection. The most vulnerable part of the house are the windows which are fragile and very easy to overcome. The glassbreak or shock detector can be used to detect the potential intruder. The goal of this paper also designs a glass-break detector which is based on the accelerometer. The accelerometer can be used as a detector which can detect the vibration in the glass panels. Final devices should be able to communicate with commercially made Control and Indicating Equipment.
\end{abstract}

\section{Introduction}

Every modern household has the system which prevents the object against the potential intruder. This system is called the Intruder Alarm System (IAS). It is part of the technical security which brings a lot of advantages that the physical security. According to the [1] "When a person stares at a screen for more than 20 minutes, his attention drops by $30 \%$; and for periods over an hour, this drop can reach $70 \%$." It means that the electronic system is much more suitable for the security than a real person. Also, the final cost is lower for the IAS that the person.

The main part of the IAS is the Control and Indicating Equipment (CIE) which is responsible for the receiving the information from the alarm detectors in the system. The author [2] mentioned that "Every CIE must be designed according to the CSN EN 50131-3 Alarm systems -Intrusion and hold-up alarm systems - Part 3: Control and indicating equipment."

However, the CIE itself does not detect any of potential intruders. The detection is done using the variation of the alarm detectors. These detectors can be divided into several areas. According to the environment, detectors can be divided into the outdoor or indoor. The author [3] stated the classification of the environment for the alarm detectors could be divided into four following categories.

- indoor

- indoor general

- outdoor protected

- outdoor general

Each category is suitable for different detectors. The main features of the detectors in a different category is mostly the temperature and the weather effects. The alarm detector in the general outdoor category must operate even if the device is exposed to the temperature below freezing or continuous rain. On the other hand, the indoor detectors are made only for the heated indoor areas where the temperature never dropped below the freezing point. The detectors can also be divided according to the part of the building where are placed. However, the most important is the peripheral which is the physical protection of the possession. The potential intruder has to overcome the wall, door or window. The strongest protection of the house is the wall. It is very difficult and also noisy to break through the wall. That is why intruders usually use the door or window. There are many security doors on the market, and the door protection is sufficient in most cases. The tricky part comes with the window protection which is the most vulnerable part of the peripheral protection.

The window protection can be improved by the special security foil which is composed of the flexible plastic. This foil can absorb the destructing force. Despite the foil, the intruder will penetrate the window without any alarm. The foil should be combined with the appropriate alarm detector. Used detector indicates the intruder and sends the alarm message to the CIE to call for help. At the same time, the foil extends the time of the penetration into the building. The most suitable detector for the windows is the glass-break detector.

The aim of this research is to design a modern glassbreak detector, which is based on the accelerometer without any acoustic verification. The final device should be able to communicate with the commercially made CIEs.

\section{Glass-break Detector}

The glass-break detector can be constructed in two different forms. The first one is based on the sound and the second is based on the vibration.

According to the author [5] "Vibration glass-break detectors employed aluminum foil conductors mounted on the inner surface of glass enclosures and sounded an alarm when the physical event of glass breaking broke

* Corresponding author: v2mach@fai.utb.cz 
the conducting path." This manner uses only the vibration in the glass. It can be distorted by the vibration which is not associated with the intruder. These vibrations can be caused by the strong wind, and the detector should not react to these subjects. The second group is based on the specified sound which is emitted by breaking glass. The author [5] stated that "The glassbreak sound signal energy is in the higher frequencies above $14 \mathrm{kHz}$ and that the onset of a glass break sound is an energetic signal." The combination of mentioned manners is used in the real detector. It has double detection using vibration and the sound effect which brings high reliability.

\section{Arduino Platform}

The Arduino platform was chosen in this paper as the microcontroller which processes the signal from the sensor. The author [6] mentioned that "The Arduino is an open-source project that started in 2005 in Interaction Design Institute as a low-cost development kit for applications with microcontrollers by Atmel. It is a physical platform with a microcontroller and several peripherals that are programmed by means of a simple development environment." The platform has several mainboards which contain of different microcontrollers and features. For the purposes of this research, the Arduino UNO was chosen due to its cost and effectiveness.

The author [6] also stated that "The Arduino UNO is based on the microcontroller Atmel ATmega328p. It is equipped with $14 \mathrm{I} / \mathrm{O}$ pins. Some of these pins support PWM, analogue inputs and/or connecting of a $16 \mathrm{MHz}$ crystal." This feature sufficiently meets the demand of this research. It also has Two Wire Interface (TWI) which can be used for the communication with other devices, in this case with the accelerometer. Arduino UNO also has the serial interface in the form of the USB.

\section{Accelerometer}

The author [7] stated that "The accelerometer being a very sensitive sensor results in a differing output even with the slightest change in orientation or placement." The accelerometer MPU-6050 was chosen for this research due its low cost, easy to mount and requires only 3-analogue connections with less power consumption, taking up to $5 \mathrm{~V}$. The chip has 6-axis device that combines a 3-axis gyroscope, 3-axis accelerometer, and a Digital Motion Processor (DMP) which process the data inside. The output of the accelerometer has 6-bit Analog to Digital Converters (ADC) for digitizing the gyroscope and accelerometer outputs. [8] These outputs can be transmitted via the USB to the computer.

The author [8] also mentioned that "DMP helps lower system power consumption by allowing the system processor to read the sensor data in bursts and then enter a low-power mode as the MPU collects more data." The chip comes on the market in custom design. It means that the chip is placed on the Printed Board
Circuit (PCB) which consists of all needed components such as voltage regulator. It also has pinheads which are necessary for the connection with the Arduino UNO. Communication with all registers of the device is performed using either TWI at $400 \mathrm{kHz}$. The PCB with the MPU-6050 can be seen in the following figure.

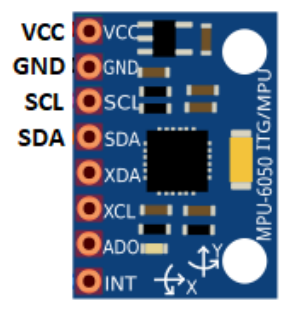

Fig. 1. The microcontroller MPU-6050

On the PCB can be found all necessary components for the proper communication. The power voltage is $5 \mathrm{~V}$; However, the MPU-6050 operates at 3.3 V. Due to this problem a voltage regulator is placed on the PCB.

\section{Main experiment}

In this experiment, the Arduino UNO was used as the main microcontroller. The Arduino has TWI interface which allows connecting MPU-6050 directly to it. The Arduino community created the library which can be used to communicate with the chip without any problems. The communication is established only by the four cables where two cables are used for the power supply, and two cables are used for the data transfer via mentioned TWI protocol. The connection can be found in the following figure.

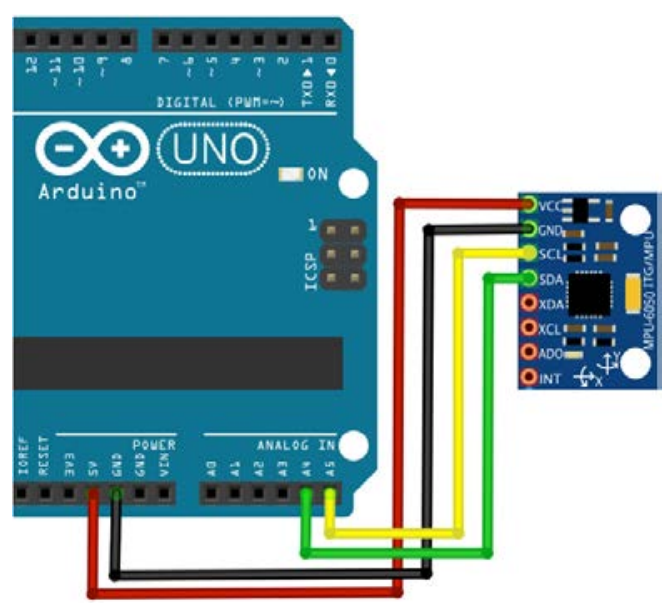

Fig. 2. Schematic of the connection.

From the schematic can be seen several unconnected pins. These additional pins can be used for different communication protocol and further function. However, for the purposes of this research, only four cables are needed. With the proper software, it is possible to obtain data from the MPU-6050. These data are in the form of the six digital numbers which are periodically transmitted by the MPU-6050. The main goal is to mount the MPU-6050 on the window and try to simulate 
its breaking. The vibration created by force can be evaluated as an intrusion. There must be set the thresholds values to avoid the vibration from the footsteps, car or the passing car. These thresholds values are listed in the chapter Results.

\section{Software Design}

As mentioned before, the MPU- 6050 has the library which can be downloaded online. There are several setting which can be chosen at the beginning of the program. These settings adjusting the format of the incoming data from the MPU-6050 and also the speed of the communication. The initialization part of the program can be found in the following figure.

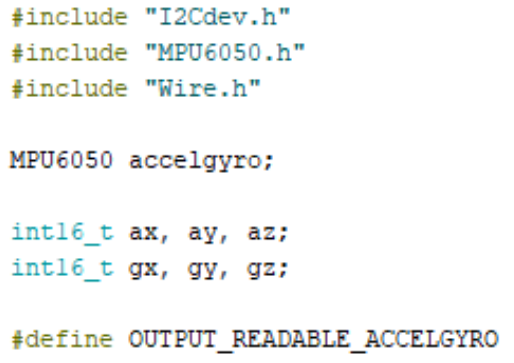

Fig. 3. Initialization program.

As mentioned before, the program must distinguish between the actual intruder and the events caused by the environment. The MPU-6050 is very sensitive even to a single step in the room. The main calibration can be found in the following chapter. The calibration is focused only on the output data.

\subsection{Calibration}

As mentioned before, the data are received in the decimal form for every axis and direction of the velocity. The full-scale range of the gyro sensors may be digitally programmed to $\pm 250, \pm 500, \pm 1000$, or \pm 2000 degrees per second. Raw data before calibration can be found in the following figure.

$\begin{array}{llllll}-8860 & -2148 & 12652 & -2808 & -852 & 1760 \\ -8732 & -2244 & 12424 & -4877 & -3170 & 2579 \\ -8996 & -2184 & 12412 & -215 & -23 & -64 \\ -8900 & -2180 & 12636 & -1 & 168 & -76 \\ -8848 & -2160 & 12584 & 51 & 327 & -88 \\ -8956 & -2144 & 12640 & 86 & 455 & -99 \\ -8716 & -2260 & 12404 & -111 & 92 & -190 \\ -8900 & -2132 & 12516 & -33 & 204 & -175\end{array}$

Fig. 4. Incoming data from the MPU-6050.

Just like any sensor, the MPU-6050 needs to be calibrated before it is used for the first time. Therefore, it needs to adjust the offsets in order to counteract zero error. The program itself is embedded in the library. The program will make an average of a few hundred readings and display the offsets required to remove zero error. Before calibration, the chip must be placed on the flat surface. All variables are calibrated, and the default positions for the gyroscope and accelerometer are represented by the zero.

\subsection{Final program}

The final program can be loaded to the microcontroller after successful calibration of the system. After successful initialization, the main request can be sent to the MPU-6050. This request asks for the actual states of all axes and all velocities. All data are automatically stored in created variables, and it can be replaced by another request. The request and the sending values via the serial interface can be found in the following figure.

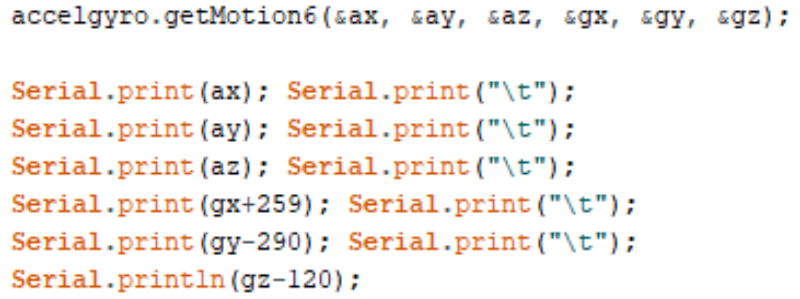

Fig. 5. Request for the data.

The previous figure also shows the function of the displaying actual calibrated values. Every possible environmental influence is simulated, and the results are converted to the thresholds levels. These level are used later in the final program evaluation. Some of the influences are listed in the following figure.

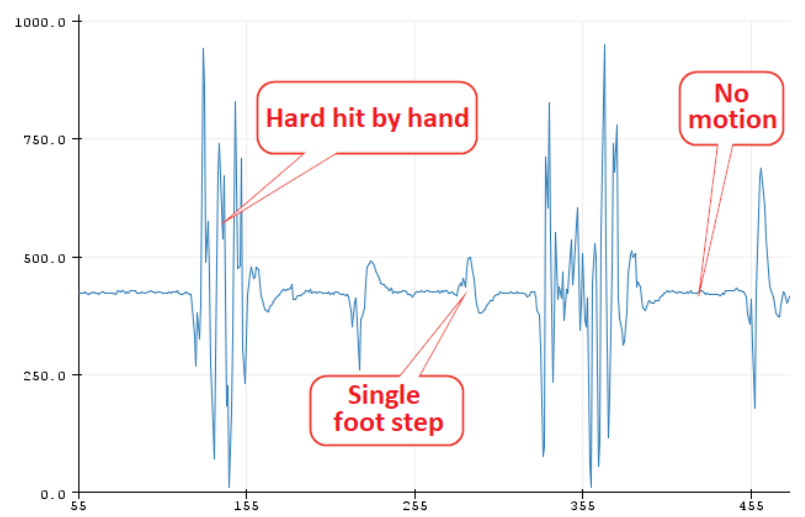

Fig. 6. Graphical visualization of the data.

The previous figure shows the visualized output of the MPU-6050 with several influences. Each state is marked and converted to the value. Every influence has its unique form, and similar signals must be distinguishable. It means that some of the incoming signals should be evaluated. The previous chapter shows the signals in the graphical interpretation for the easy explanation. However, the incoming signal is in the decimal form which is more suitable for the final evaluation.

Only one axis can be used for the evaluation because when the window is hit, the energy is acting only in one direction. When the chip is placed on the window, the evaluated force is the acceleration in the y-axis. The basic evaluation is done only by comparison of the incoming signal and the referenced one. 
The system should also have the relay which can be used as alarm trigger. This patter is used in many alarm applications. The alarm trigger can also be created by the communication via serial interface where several detectors can be connected to just one digital bus. The final product should have signalling diode for the verification. The main part of the detector is MPU-6050 itself. It means, that accelerometer can be mounted on the window and the Arduino UNO can be placed elsewhere. The communication is provided by the TWI which allows connecting several accelerometers in just one Arduino UNO. This can be used in the place with many windows, and this concept is also cost-effective.

\section{Results}

This work was focused on the alternative design for the glass-break detector. The standard construction of the detectors was replaced by the standard accelerometer. The accelerometer was able to detect an even small amount of vibration which can be produced by the potential intruder. The final system is composed of the accelerometer MPU-6050 with the Arduino UNO. This concept also provides the cost efficiency because to just one Arduino UNO can be connected several accelerometers which can communicate on one bus.

The experiment proved that the accelerometer should be used as alarm detector mounted on the window. This application is more accurate than any commercially made glass-break detectors, and it can prevent possible false alarms. Further research can be focused on the examination by comparing the waveforms of the signals.

This work was supported by the Ministry of Education, Youth and Sports of the Czech Republic within the National Sustainability Programme Project No. LO1303 (MSMT7778/2014) and also by the European Regional Development Fund under the project CEBIA-Tech No. CZ.1.05/2.1.00/03.0089 and by the Internal Grant Agency of Tomas Bata University under the project No. IGA/CebiaTech/2018/004.

\section{References}

1. J. Landa, Ch. Jun, M. Jun. ICMTMA, (2017)

2. J. Valouch. AMM. (2015)

3. V. Mach. T. 5(2), (2016)

4. M. Lojka, M. Pleva, E. Kiktová, J. Juhár, A. Čižmár. MTA. 75(17), (2016)

5. B. Gestner J. Tanner, D. Anderson. ISCS, (2007)

6. M. Pospisilik T. Smekal, M. Adamek, P. Neumann, T. Dulik. CSCC. (2016)

7. A. Kannan, A. Ramesh, L. Srinivasan V. Vijayaraghavan. GIoTS (2017)

8. S. Umbarkar, G. Rajput, S. Halder, P. Harname, S. Mendgudle. ICCASP. (2017). 